\title{
Radon - un risque sous-estimé dans l'habitat
}

\author{
Le radon provient de la désintégration de l'uranium dans le sol. Ce gaz naturel radio- \\ actif provoque chaque année en Suisse 200 à 300 décès par cancer du poumon. Des \\ techniques de construction permettent de réduire considérablement la concentra- \\ tion de radon dans les bâtiments neufs et rénovés. Il est important que les profes- \\ sionnels de la médecine, de la santé publique, de la science et du secteur du bâtiment \\ s'échangent sur la protection du radon.
}

Patrizia Frei

Responsable projet Environnement et tabac, Ligue suisse contre le cancer

Correspondance:

Dr phil. nat. Patrizia Frei

Ligue suisse contre le cancer Effingerstrasse 40

CH-3001 Berne

Tél. 0313899206

journeecancer[at]liguecancer.ch
Le gaz radioactif radon est naturellement présent dans le sol; ses émanations dans l'air sont invisibles, inodores et insipides. À l'air libre, il ne présente aucun risque pour la santé. Il peut cependant s'infiltrer par les défauts d'étanchéité de l'enveloppe des bâtiments et s'accumuler dans l'air ambiant. Lors de la respiration, les produits de désintégration du radon se déposent sur le tissu pulmonaire et l'irradient. Une concentration croissante de radon augmente ainsi le risque de contracter un cancer du poumon.

\section{Cancer du poumon engendré par le radon}

Les résultats de nouvelles études épidémiologiques réalisées dans l'habitat montrent que le risque, extrapolé à partir de données sur les travailleurs de mines, a jusqu'ici été sous-évalué [1]. Sur la base de ces conclusions, l'Organisation mondiale de la santé a recommandé en 2009 une concentration maximale de $300 \mathrm{~Bq} / \mathrm{m}^{3}$, afin de réduire les risques sanitaires liés au radon dans l'habitat [2].

Selon cette évaluation récente du risque, toutes les régions de Suisse sont désormais concernées par la

\section{Journée du cancer 2014}

La Ligue suisse contre le cancer organisera une journée sur le thème du radon le 4 décembre 2014 à l'Hôtel National de Berne, en collaboration avec l'Office fédéral de la santé publique et en partenariat avec la Société suisse des ingénieurs et des architectes (SIA).

Des intervenants spécialisés dans les domaines de la médecine, de la science et du bâtiment apporteront chacun leur éclairage sur les risques liés au radon. Cette journée s'adresse aux professionnels de la médecine, de la santé publique et de la construction, aux scientifiques, aux personnes intéressées, ainsi qu'aux représentants des autorités, aux propriétaires immobiliers et aux notaires. Elle se terminera par un débat public auquel participeront, entre autres, le Dr Roland Charrière, directeur suppléant de l'Office fédéral de la santé publique et le Prof. Jakob Passweg, président de la Ligue suisse contre le cancer.

Vous trouverez le programme de la Journée du cancer 2014, les modalités d'inscription et des informations complémentaires sous www.liguecancer.ch/ journeeducancer.

problématique du radon, et plus seulement les régions à risque élevé des Alpes et de l'Arc jurassien définies jusqu'ici. Le radon génère huit à dix pourcent des décès par cancer du poumon, soit 200 à 300 cas par année en Suisse. Ce chiffre est comparable à celui des décès liés aux mélanomes. Le radon est la cause la plus fréquente de cancer du poumon après le tabagisme; pour les non-fumeurs, il est le plus grand facteur de risques.

\section{Protection contre le radon en Suisse}

En 2011, l'Office fédéral de la santé publique a lancé le Plan d'action radon 2012-2020, dans le but d'assurer la protection de la population [3]. L'élément-clé de ce plan d'action est la révision de la législation sur la radioprotection, dont les valeurs légales doivent être ajustées. Promouvoir la protection contre le radon dans le secteur du bâtiment est également un élément central: Des techniques appropriées lors de la construction et des transformations permettent de réduire considérablement et facilement la concentration de radon dans les bâtiments. Toute la population suisse peut être exposée à des concentrations accrues de radon. La protection contre ce gaz est par conséquent nécessaire.

\section{Références}

1 Darby S, Hill D, Deo H, Auvinen A, Barros-Dios JM, Baysson $\mathrm{H}$, et al. Residential radon and lung cancer detailed results of a collaborative analysis of individual data on 7148 persons with lung cancer and 14208 persons without lung cancer from 13 epidemiologic studies in Europe. Scand J Work Environ Health. 2006;32 suppl 1:1-84.

2 World Health Organisation (WHO). WHO handbook on indoor radon: a public health perspective. www.who.int/ionizing_radiation/env/radon/en/ index1.html. 2009.

3 Office fédéral de la santé publique (OFSP). Plan d'action radon 2012-2020. http://www.bag.admin.ch/ themen/strahlung/00046/11649/index.html?lang=fr. 2011. 